\title{
Quantum-statistical properties of a Raman-type model
}

\author{
Alexander S. Shumovsky* and B. Tanatar \\ Department of Physics, Bilkent University, Bilkent, 06533 Ankara, Turkey
}

(Received 10 May 1993; revised manuscript received 21 July 1993)

\begin{abstract}
A model describing three boson fields with the decay of Rayleigh mode into the Stokes and vibration (phonon) modes is examined. The problem of exact eigenvalues and eigenstates is reduced to the calculation of zeros of new orthogonal polynomials defined in terms of difference and differential equations. The instability of the spectrum of eigenvalues is established. The quantum-statistical properties are investigated for various initial conditions. The possibility of using the correlation Raman spectroscopy to measure the quantum-statistical properties of the vibration mode is discussed.

PACS number(s): 42.50.-p, 42.65.-k, 32.80.Bx
\end{abstract}

\section{INTRODUCTION}

Raman scattering is known as an important method of spectroscopy of molecules and solids [1]. The standard measurements of frequency and angular distribution of scattered light give us very important information about the linear properties of vibration (phonon) modes. A more detailed picture would arise from the measurement of quantum distribution function or of higher-order correlation functions of phonons. It should be emphasized that this distribution can differ markedly from the BoseEinstein distribution for bosons even at equilibrium with a given temperature $T[2]$. An essential physical quantity that may be measured by the methods of correlation spectroscopy [3] is the degree of coherence of second-order for the scattered light. However, it is necessary to know how the statistical properties of scattered light are related to the statistical properties of the vibration mode.

The recent progress in quantum optics permits us to discuss the possibility of using the nonclassical states of light as the pumping field in a Raman scattering process. It is interesting to compare the changes in statistical properties of scattered light with the changes in the pumping field at a given state of the vibration mode.

To solve these problems it is necessary to examine a full quantum model of the Raman scattering. The simplest model of such type is described by the Hamiltonian [4]

$H=\omega a^{\dagger} a+\omega_{S} a_{S}^{\dagger} a_{S}+\omega_{b} b^{\dagger} b+\gamma\left(b^{\dagger} a_{S}^{\dagger} a+a^{\dagger} a_{S} b\right)$,

where $a^{\dagger}$ and $a_{S}^{\dagger}$ are the creation operators for the Rayleigh and Stokes modes, respectively, with the corresponding frequencies $\omega$ and $\omega_{S}, b^{\dagger}$ is the creation operator for the vibration mode with frequency $\omega_{b}$, and $\gamma$ is the coupling constant. We restrict our consideration to the Stokes process only because we will examine the case of low intensity initial field. We note that the same form of the Hamiltonian is usually employed in the theory of parametric optical processes [5-8].

\footnotetext{
*Permanent address: Bogolubov Laboratory of Theoretical Physics, JINR, Dubna, Moscow Region, Russia.
}

The change of operators $a^{\dagger}$ and $a$ by $c$ numbers, corresponding to the classical amplitudes of an intense laser field, [9] leads to an effective bilinear boson Hamiltonian. In this case the problem can be solved exactly [10] with the aid of Bogolubov canonical transformation [11]. But of course, it is impossible to speak about the quantumstatistical properties of the Rayleigh field in such an approach.

Another type of effective Hamiltonian is connected with an analogy in mathematical formulation of Raman scattering process and of the interaction of a set of twoor three-level atoms with the resonant radiation fields [12-15]. This problem is also solved exactly in the single atom case [16]. The model systems associated with the Hamiltonian of Eq. (1) have been examined using various techniques, such as numerical solution $[17,18]$, operator linearization method [19], and the short-time approximation [20]. The Heisenberg dynamical equations have been solved exactly [21] by applying some iterative procedure. The existence of simple conservation laws can also be used to construct the exact solution in the Schrödinger picture $[2,22,23]$. The exact eigenvalues of Eq. (1) can be expressed in terms of new orthogonal polynomials $[2,22]$ which are reduced to the Hermite polynomials in some special case.

In the present paper we use the exact solution of the problem with the Hamiltonian of Eq. (1) in the Schrödinger representation $[2,22]$ in order to examine the quantum-statistical properties of scattered light and its dependence on the properties of Rayleigh field and phonons.

The organization of this paper is as follows. In Sec. II we present the details of our exact solution of the trilinear boson Hamiltonian for the Raman-type model. We discuss the structure of the eigenvalue spectrum and the corresponding eigenstates, and their asymptotic behavior. We then describe in Sec. III the dynamics of the Raman-type model considered for different preparations of the photon and phonon initial state. In Sec. IV we exhibit our numerical results of the dynamical problem for various initial photon and phonon distributions. Discussion of our results in connection with other approaches and experiments is given. Finally, we conclude with a 
brief summary stressing the physical significance of our calculations.

\section{EIGENVALUES AND EIGENSTATES}

In this section we investigate the eigenvalue spectrum and eigenstates of the Hamiltonian of Eq. (1) with the assumption of the exact resonance condition

$$
\omega=\omega_{S}+\omega_{b} .
$$

It follows from the boson commutation relations that the Hamiltonian depicted in Eq. (1) has the following integrals of motion:

$$
a^{\dagger} a+a_{S}^{\dagger} a_{S}=\text { const }, \quad a^{\dagger} a+b^{\dagger} b=\text { const },
$$

which express the Manley-Rowe relations in nonlinear optics [1]. From these two conservation laws we construct the following operator:

$N=a^{\dagger} a+\left(a_{S}^{\dagger} a_{S}+b^{\dagger} b\right) / 2$ such that $[N, H]=0$,

which describes the number of collective excitations in the system. Thus, we can consider the eigenfunctions of $N$

$$
N\left|\psi^{(n)}\right\rangle=n\left|\psi^{(n)}\right\rangle
$$

as the eigenstates of Hamiltonian given by Eq. (1) corresponding to the $n$th excited state

$$
H\left|\psi^{(n)}\right\rangle=E^{(n)}\left|\psi^{(n)}\right\rangle,
$$

with the eigenvalue $E^{(n)}$. It follows from the definition of Eq. (4) that $\left|\psi^{(n)}\right\rangle$ should be chosen in the following form:

$$
\left|\psi^{(n)}\right\rangle=\sum_{j=0}^{n} \lambda_{j m}^{(n)}|n-j, j, m+j\rangle \text { for all } n
$$

with

$$
\sum_{j=0}^{n}\left|\lambda_{j m}^{(n)}\right|^{2}=1
$$

where $|k, l, p\rangle$ is the direct product of corresponding number states for Rayleigh, Stokes, and vibration modes. Then, for any $n$ the eigenvalues $E^{(n)}$ are determined by the equation

$$
\operatorname{det} X^{(n)}=0,
$$

where $X^{(n)}$ is the real symmetric $(n+1) \times(n+1)$ matrix with elements

$$
X_{\alpha \beta}^{(n)}= \begin{cases}-x^{(n)} & \text { if } \alpha=\beta \\ {[(n-\alpha+1) \alpha(m+\alpha)]^{1 / 2}} & \text { if } \beta=\alpha+1 \\ 0 & \text { otherwise. }\end{cases}
$$

In the above expression we have

$$
x^{(n)}=\left(E^{(n)}-\omega n-\omega_{b} m\right) / \gamma .
$$

The recursion relation between the coefficients of wave function of Eq. (7) has the form

$$
\begin{aligned}
\lambda_{j+1}^{(n)}[ & (n-j)(j+1)(m+j+1)]^{1 / 2} \\
& =x^{(n)} \lambda_{j}^{(n)}-\lambda_{j-1}^{(n)}[(n-j+1) j(m+j)]^{1 / 2} .
\end{aligned}
$$

This recursion relation will be represented by the equivalent expression

$$
P_{j+1}^{(n)}(x)=x P_{j}^{(n)}(x)-q_{j}^{(n)} P_{j-1}^{(n)}(x),
$$

defining the new orthogonal polynomials $P_{j}^{(n)}(x)$, which have been previously introduced $[22,23]$ for $m=0$. Here

$$
\begin{aligned}
& P_{j}^{(n)}(x)=\xi_{j}^{(n)} \lambda_{j}^{(n)}(x), \\
& \xi_{j+1}^{(n)}=\xi_{j}^{(n)}[(n-j) j(m+j+1)]^{1 / 2}, \\
& q_{j}^{(n)}=(n-j+1) j(m+j) .
\end{aligned}
$$

Then instead of Eq. (8), we obtain

$$
P_{n+1}^{(n)}(x)=0
$$

which is the equation for the eigenvalues of Hamiltonian given in Eq. (1). For any fixed $n$ this equation has $n+1$ real roots.

In addition to the difference equation given in Eq. (12), the orthogonal polynomials $P_{j}$ can be defined also by the differential equation [23]

$$
x F(x, t)=\frac{\partial F}{\partial t}+t\left(n(m+1) \frac{\partial}{\partial t}-t \frac{\partial^{2}}{\partial t^{2}}\right)(t F),
$$

where the generating function has the form

$$
F(x, t)=\sum_{k=0}^{\infty} P_{k}(x) \frac{t^{k}}{k !} .
$$

The polynomials $P_{j}(x)$ in turn can be expressed in terms of the Bernoulli polynomials $B_{l}^{j+1}(n)$ in the following way:

$$
P_{j}^{(n)}=\sum_{l=0}^{j} \varphi_{l j}^{(n)} x^{l}
$$

where

$$
\varphi_{l, j+1}^{(n)}=\beta_{l}^{j+1}(n) B_{l}^{j+1}(n)
$$

and

$$
\begin{aligned}
& \beta_{l}^{j+1}(n)(1-l / j)=-q_{j}^{(n)} \beta_{l}^{j-1}(n), \\
& \beta_{l}^{j+1}(n) l(n / j-1)=\beta_{l-1}^{j}(n) .
\end{aligned}
$$

With the help of the above relations, we obtain for the coefficients of the eigenfunctions given in Eq. (7)

$$
\lambda_{j}^{(n)}\left(x_{i}\right)=\lambda_{0}^{(n)}\left(x_{i}\right) \frac{P_{j}^{(n)}\left(x_{i}\right)}{F_{j}^{(n)}}
$$


where $x_{i}$ is any solution of Eq. (8) [or of the equivalent Eq. (13)] and

$$
F_{j}^{(n)}=\left[(j !)^{3}\left(\begin{array}{c}
n \\
j
\end{array}\right)\left(\begin{array}{c}
m+j \\
j
\end{array}\right)\right]^{1 / 2}
$$

The coefficient $\lambda_{0}^{(n)}\left(x_{i}\right)$ is determined from the normalization condition

$$
\sum_{j=0}^{n}\left|\lambda_{j}^{(n)}\left(x_{i}\right)\right|^{2}=1 \text { for all } x_{i}, \quad i=1,2, \ldots, n+1
$$

For small $n$, the coefficients given by Eq. (14) as well as the eigenvalues $E^{(n)}$ can be calculated analytically. We list a few of those in Table I.

One can observe from Eq. (13) that the roots $\left\{x_{j}^{(n)}\right\}$ are ordered symmetrically with respect to zero (we have enumerated the roots starting from the maximum value). The value $x^{(n)}=0$ is the root of Eq. (13) for even $n$ only. It means that for $n=2 k$, in the spectrum of eigenenergies $E^{(n)}$ there exists a central line with energy $E_{k+1}^{(2 k)}=2 k \omega+$ $m \omega_{b}$, while for $n=2 k+1$ such a line is absent in the spectrum.

Using the Hadamard criterion [24], it is not difficult to show that the maximum root has the following asymptotic behavior

$$
x_{\max } \sim \begin{cases}n^{3 / 2}, & n \rightarrow \infty, \quad m \ll n \\ m^{1 / 2} n, & m \rightarrow \infty, \quad n \ll m\end{cases}
$$

It follows from the symmetry of roots that $x_{\min }=-x_{\max }$. Thus, the minimum eigenvalue has the asymptotic behavior
$E_{\min } \simeq n \omega+m \omega_{b}-\gamma x_{\max } \sim n \omega+m \omega_{b}-\gamma\left[n^{3}+\alpha n^{2} m\right]^{1 / 2}$.

From Eq. (15) we observe that for any fixed $\omega, \omega_{b}$, and $\gamma$ it is possible to find $n_{0}$ such that for any $n>n_{0}$, the value of $E_{\min }$ becomes negative. In other words, we have instability of eigenvalues of the Hamiltonian given in eq. (1) with respect to the number of photons in the Rayleigh mode, whereas they are stable relative to the number of excitations in the vibration mode.

It is not surprising to find such an instability for the system with a cubic nonlinearity. Similar results occur for the system describing the decay of a mode of Bose fields into $p$ modes with $p \geq 2[2,22]$. The possible physical reasons for such an instability have been discussed in detail in Ref. [22].

\section{DYNAMICAL BEHAVIOR}

If we know the set of eigenvalues and eigenfunctions for any $n$ and $m$, the time dependent wave function is represented by

$$
|\psi(t)\rangle=\sum_{n, m=0}^{\infty} \sum_{l=1}^{n+1} C_{l m}^{(n)} \exp \left(-i E_{l}^{(n)} t\right)\left|\psi_{l}^{(n)}\right\rangle,
$$

where the index $l$ enumerates the roots of Eq. (8) and coefficients $C_{l m}^{(n)}$ are determined by the initial conditions. We have suppressed the dependence on $m$ of the eigenvalues $E_{l}^{(n)}$ and the coefficients $\lambda_{j l}^{(n)}$ in the expansion of $\left|\psi_{l}^{(n)}\right\rangle$ determined by the relations of Eqs. (13) and (14).

TABLE I. List of the orthogonal polynomials $P_{l}^{(n)}(x)$, zeros of these polynomials, the eigenvalues $E^{(n)_{l}}$, and the coefficients for eigenstates $\lambda_{l}^{(n)}$ for $n=0,1$, and 2 , and fixed $m$.

\begin{tabular}{l|l}
\hline \hline$n=0$ & $P_{1}^{(0)}(x)=x$ \\
& $x_{1}=0$ \\
& $E_{0}^{(0)}=m \omega_{b}$ \\
& $\lambda_{0}^{(0)}\left(x_{1}\right)=1$ \\
& $P_{2}^{(1)}(x)=x^{2}-(m+1)$ \\
& $x_{1}=\sqrt{m+1}, \quad x_{2}=-\sqrt{m+1}$ \\
& $E_{1}^{(1)}=\omega+m \omega_{b}+\gamma \sqrt{m+1}$ \\
& $\lambda_{0}^{(1)}\left(x_{1}\right)=\frac{1}{\sqrt{2}}, \lambda_{1}^{(1)}\left(x_{1}\right)=\frac{1}{\sqrt{2}}$ \\
& $E_{2}^{(1)}=\omega+m \omega_{b}-\gamma \sqrt{m+1}$ \\
& $\lambda_{0}^{(1)}\left(x_{2}\right)=\frac{1}{\sqrt{2}}, \quad \lambda_{1}^{(1)}\left(x_{2}\right)=-\frac{1}{\sqrt{2}}$ \\
& $P_{3}^{(2)}(x)=x^{3}-x(4 m+6)$ \\
& $E_{1}^{(2)}=2 \omega+m \omega_{b}+\gamma \sqrt{4 m+6}$ \\
& $\lambda_{0}^{(2)}\left(x_{1}\right)=\left(\frac{m+1}{4 m+6}\right)^{1 / 2}, \quad \lambda_{1}^{(2)}\left(x_{1}\right)=\frac{1}{\sqrt{2}}, \quad \lambda_{2}^{(2)}\left(x_{1}\right)=\left(\frac{m+2}{4 m+6}\right)^{1 / 2}$ \\
& $E_{2}^{(2)}=2 \omega+m \omega_{b}$ \\
& $\lambda_{0}^{(2)}\left(x_{2}\right)=\left(\frac{m+2}{2 m+3}\right)^{1 / 2}, \quad \lambda_{1}^{(2)}\left(x_{2}\right)=0, \quad \lambda_{2}^{(2)}\left(x_{2}\right)=-\left(\frac{m+1}{2 m+3}\right)^{1 / 2}$ \\
& $E_{3}^{(2)}=2 \omega+m \omega_{b}-\gamma \sqrt{4 m+6}$ \\
& $\lambda_{0}^{(2)}\left(x_{3}\right)=\left(\frac{m+1}{4 m+6}\right)^{1 / 2}, \quad \lambda_{1}^{(2)}\left(x_{3}\right)=-\frac{1}{\sqrt{2}}, \quad \lambda_{2}^{(2)}\left(x_{3}\right)=\left(\frac{m+2}{4 m+6}\right)^{1 / 2}$ \\
\hline \hline
\end{tabular}


Taking into account of the explicit form of the eigenvalues

$$
E_{l}^{(n)}=n \omega+m \omega_{b}+\gamma x_{l m}^{(n)}
$$

where $\left\{x_{l m}^{(n)}\right\}$ are the solutions of Eq. (13), symmetric relative to $x^{(n)}=0$, we obtain for the wave function

$$
|\psi(t)\rangle=\sum_{n, m=0}^{\infty} e^{-i\left(n \omega+m \omega_{b}\right) t} \sum_{l=1}^{n+1} C_{l m}^{(n)} e^{-i \gamma x_{l m}^{(n)} t}\left|\psi_{l}^{(n)}\right\rangle .
$$

Then the time dependent average of any dynamical variable $\hat{A}$ of the system under consideration is determined by

$$
\langle\hat{A}\rangle_{t}=\langle\psi(t)|\hat{A}| \psi(t)\rangle
$$

We shall consider the first- and second-order correlation functions for the Rayleigh and Stokes photons in the system. For the mean number of Rayleigh photons we get

$$
\begin{aligned}
\left\langle a^{\dagger} a\right\rangle_{t}=\sum_{n, m=0}^{\infty} \sum_{k, l=1}^{n+1} & C_{k m}^{(n) *} C_{l m}^{(n)} e^{-i \gamma\left(x_{k m}^{(n)}-x_{l m}^{(n)}\right) t} \\
& \times \sum_{j=0}^{n} \lambda_{j k m}^{(n) *} \lambda_{j l m}^{(n)}(n-j) .
\end{aligned}
$$

Then, according to the Manley-Rowe relations (cf. Sec. I) we have for the Stokes photons

$$
\left\langle a_{S}^{\dagger} a_{S}\right\rangle_{t}=\left\langle a^{\dagger} a\right\rangle_{0}-\left\langle a^{\dagger} a\right\rangle_{t} .
$$

For the mean squared intensities we obtain

$$
\begin{gathered}
\left\langle\left(a^{\dagger} a\right)^{2}\right\rangle_{t}=\sum_{n, m=0}^{\infty} \sum_{k, l=1}^{n+1} C_{k m}^{(n) *} C_{l m}^{(n)} e^{-i \gamma\left(x_{k m}^{(n)}-x_{l m}^{(n)}\right) t} \\
\times \sum_{j=0}^{n} \lambda_{j k m}^{(n) *} \lambda_{j l m}^{(n)}(n-j)^{2} \\
\left\langle\left(a^{\dagger} a_{S}\right)^{2}\right\rangle_{t}=\sum_{n, m=0}^{\infty} \sum_{k, l=1}^{n+1} C_{k m}^{(n) *} C_{l m}^{(n)} e^{-i \gamma\left(x_{k m}^{(n)}-x_{l m}^{(n)}\right) t} \\
\times \sum_{j=0}^{n} \lambda_{j k m}^{(n) *} \lambda_{j l m}^{(n)} j^{2}
\end{gathered}
$$

for the Rayleigh and Stokes photons, respectively. It follows from the relations of Eqs. (19)-(22) that the expectation values of number and square number of photons in both modes are independent of the mode frequencies. In other words, their dynamics is determined by the coupling constant $\gamma$. This is also the case for any expectation value of the type

$$
\left\langle\left(c^{\dagger} c\right)^{r}\right\rangle_{t}, \quad r=1,2, \ldots,
$$

where $c$ is defined to be the operator corresponding to Rayleigh, Stokes, or phonon mode. Thus, the statistical properties of scattered light are independent of the mode frequencies. The real magnitudes of expectation values are provided by the symmetry of solutions of Eq. (13).
Let us now discuss the possible choice of initial conditions. We shall consider the case when the Stokes mode is initially in the vacuum state $|0\rangle_{S}$. Let the initial states of Rayleigh $|R\rangle$ and vibration $|V\rangle$ modes be defined by the expansions

$$
\begin{aligned}
& |R\rangle=\sum_{n=0}^{\infty} p_{n}|n\rangle, \\
& |V\rangle=\sum_{m=0}^{\infty} g_{m}|m\rangle_{b} .
\end{aligned}
$$

Then the coefficients $C_{l m}^{(n)}$ in Eq. (17) are determined by the equations

$$
\sum_{l=1}^{n+1} C_{l m}^{(n)} \lambda_{j l}^{(n)}= \begin{cases}p_{n} q_{m}, & j=0 \\ 0 & \text { otherwise } .\end{cases}
$$

The distribution for the Rayleigh mode will be chosen by the following cases: (a) a number state with a given $n$

$$
p_{n^{\prime}}=\delta_{n n^{\prime}},
$$

(b) a coherent state with parameter $\alpha$

$$
p_{n}=\frac{\alpha^{n}}{\sqrt{n !}} \exp \left(-|\alpha|^{2} / 2\right),
$$

and (c) a squeezed vacuum state with parameters $\mu$ and $\nu$

$$
p_{n}=\frac{1}{\sqrt{n ! \mu}}\left(\frac{\nu}{2 \mu}\right)^{n / 2} H_{n}(0)
$$

where $H_{n}(x)$ is the Hermite polynomial of order $n$. For the vibrational mode, we shall examine the following possibilities: (a) a number state with a given $m$

$$
g_{m^{\prime}}=\delta_{m m^{\prime}},
$$

and (b) a squeezed vacuum state with parameters $\mu_{V}$ and $\nu_{V}$

$$
g_{m}=\frac{1}{\sqrt{m ! \mu_{V}}}\left(\frac{\nu_{V}}{2 \mu_{V}}\right)^{m / 2} H_{m}(0)
$$

Relating to our choice of the initial states of the Rayleigh and vibrational modes, we make the following remarks. The coherent and squeezed states of photon initial states correspond to the idea of application of a nonclassical light in the Raman scattering process. It also enables us to examine the dependence of statistical properties of scattered light both on the type of initial state of photons and that of the vibrational mode (phonons). Use of a squeezed vacuum state for the vibrational mode, on the other hand, corresponds to the phonons of a polaritonlike system for which the number distribution is given by a squeezed state at zero temperature [2].

\section{RESULTS AND DISCUSSION}

The mean values for the number of photons and their variances are normalized with respect to the initial number of Rayleigh mode photons at $t=0,\left\langle a^{\dagger} a\right\rangle_{0}$, viz. 


$$
\frac{\left\langle a^{\dagger} a\right\rangle_{t}}{\left\langle a^{\dagger} a\right\rangle_{0}}, \frac{v_{t}\left(a^{\dagger} a\right)}{\left\langle a^{\dagger} a\right\rangle_{0}}
$$

In Fig. 1, we show the normalized average number of photons (solid line) and the variance (dotted line) for the Rayleigh mode as a function of the dimensionless time variable $\gamma t$. Initially the Rayleigh mode is in the number state with $n=2$ and $n=10$ photons, depicted in upper and lower figures, respectively, in Figs. 1(a) and $1(b)$. The vibrational mode is also in the number state, indicated by $m=2$ phonons, in Fig. 1(a). For the
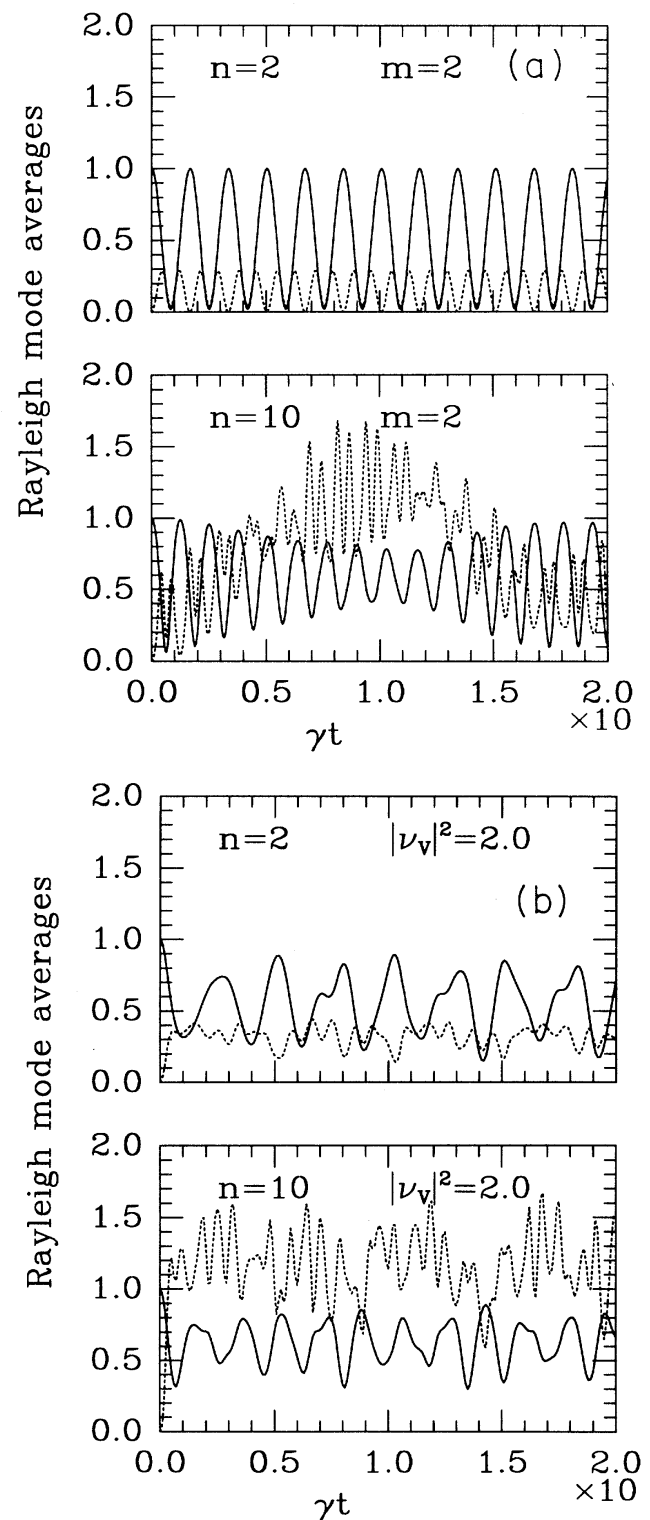

FIG. 1. The time dependence of the (normalized) average number of photons $\left\langle a^{\dagger} a\right\rangle_{t}$ (solid line) and the variance $v_{t}\left(a^{\dagger} a\right)$ (dotted line) for the Rayleigh mode initially in the number state characterized by (a) $n=2$ (upper) and $n=10$ (lower) photons and $m=2$ phonons; (b) $n=2$ (upper) and $n=$ 10 (lower) photons and in the squeezed state phonons with $\left|\nu_{V}\right|^{2}=2.0$. In both cases, the Stokes mode is initially in the vacuum state.
Stokes mode, we assume a vacuum state at $t=0$, i.e., there are no inelastic photons initially. One may observe that there is a periodical change of sign in the difference $v_{t}\left(a^{\dagger} a\right)-\left\langle a^{\dagger} a\right\rangle_{t}$, which shows the deviation from the Poissonian distribution of photons in the Rayleigh line. This deviation increases with increasing $n$ (number of photons in the Rayleigh mode) at fixed $m$ (number of phonons in the vibration mode) and decreases with increasing $m$ at fixed $n$. For $m \gg n$, the deviation vanishes and the Rayleigh mode is in a sub-Poissonian state for all later times. This fact may be illustrated by the exact solution of the dynamical problem when $n=2$ for which we have
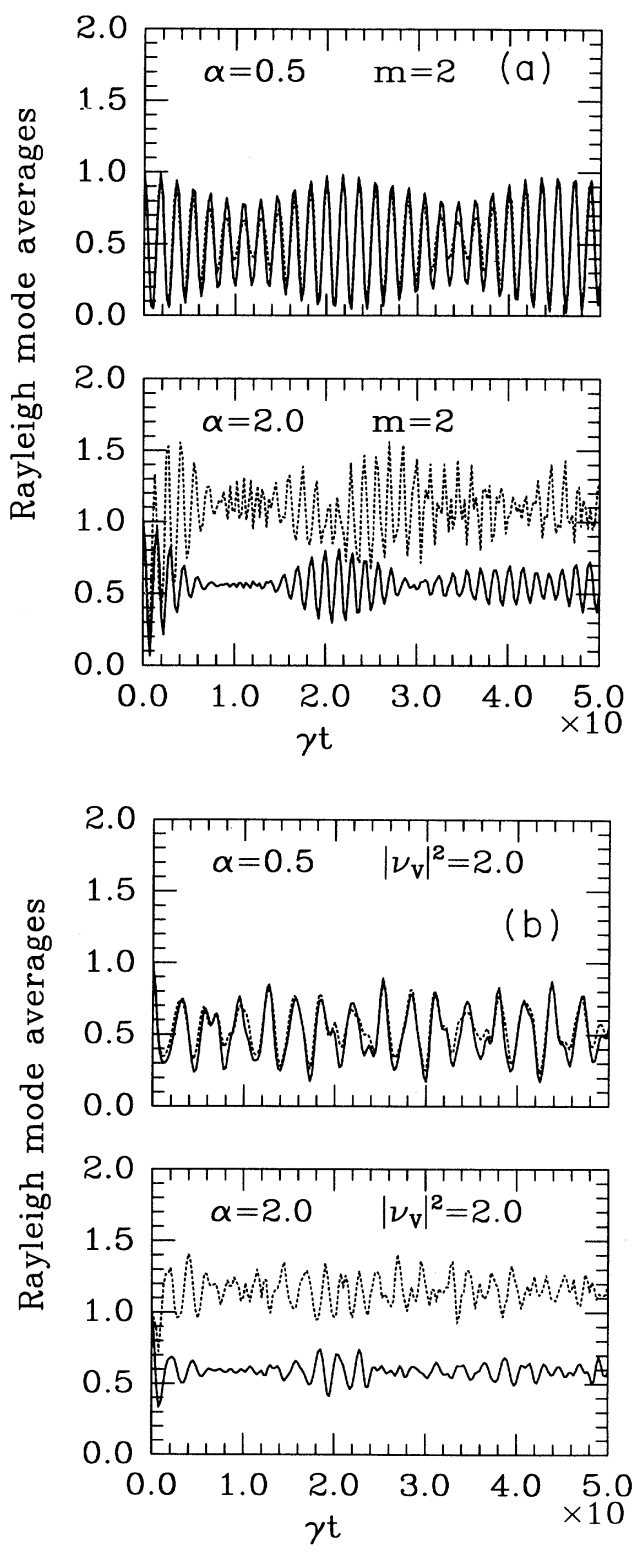

FIG. 2. Same as Fig. 1 for the Rayleigh mode initially in the coherent state characterized by (a) $\alpha=0.5$ (upper) and $\alpha=2$ (lower) photons and $m=2$ phonons; (b) $\alpha=0.5$ (upper) and $\alpha=2$ (lower) photons and in the squeezed state phonons with $\left|\nu_{V}\right|^{2}=2$. 


$$
\begin{aligned}
& \left\langle a^{\dagger} a\right\rangle_{t}=\frac{1}{(2 m+3)^{2}}\left\{2\left[(m+1) \cos \Omega_{m} t+(m+2)\right]^{2}+(m+1)(2 m+3) \sin ^{2} \Omega_{m} t\right\} \\
& \approx 1+\cos (2 \gamma \sqrt{m} t) \\
& \left\langle\left(a^{\dagger} a\right)^{2}\right\rangle_{t}=\frac{1}{(2 m+3)^{2}}\left\{4\left[(m+1) \cos \Omega_{m} t+(m+2)\right]^{2}+(m+1)(2 m+3) \sin ^{2} \Omega_{m} t\right\} \\
& \approx[\cos (2 \gamma \sqrt{m} t)+1]^{2}+\frac{1}{2} \sin ^{2}(2 \gamma \sqrt{m} t)
\end{aligned}
$$

where $\Omega_{m}=\gamma[2(2 m+3)]^{1 / 2}$, so that

$$
v_{t}\left(a^{\dagger} a\right)-\left\langle a^{\dagger} a\right\rangle_{t} \approx-\frac{1}{2}[1+\cos (2 \gamma \sqrt{m} t)]^{2}
$$

Similar time dependent behavior for $m \gg n$ was also obtained by Drobny and Jex [25]. Increasing $n$ at fixed $m$ implies an increase in the number of terms with different frequencies in the sums of Eqs. (19) and (21). Therefore, it is not surprising to observe the collapse-revival patterns as in the Jaynes-Cummings model [26,27]. We note that in Ref. [25] such time dependent behavior was examined only for the coherent initial state of the Rayleigh mode.

When the phonons are initially in the squeezed vacuum state [depicted in Fig. 1(b)] we also have change in the type of number distribution in time, but in contrast to the previous case the increase of $n$ at fixed mean number of phonons $\left\langle b^{\dagger} b\right\rangle_{0}=\left|\nu_{V}\right|^{2}$ leads to the almost superPoissonian state for both the Rayleigh and Stokes photons. As $\left|\nu_{V}\right|^{2}$ becomes larger for fixed $n$, we also observe a chiefly super-Poissonian state. In other words, we have

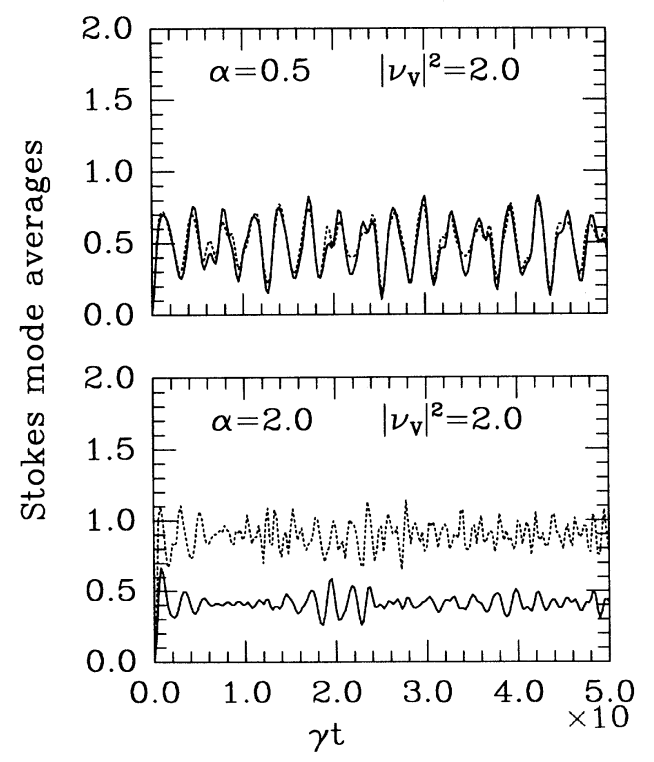

FIG. 3. The time dependence of the (normalized) average number of photons $\left\langle a_{S}^{\dagger} a_{S}\right\rangle_{t}$ (solid line) and the variance $v_{t}\left(a_{S}^{\dagger} a_{S}\right)$ (dotted line) for the Stokes mode initially in the coherent state characterized by $\alpha=0.5$ (upper) and $\alpha=2.0$ (lower) photons and in the squeezed state phonons with $\left|\nu_{V}\right|^{2}=2$. a qualitative change in the behavior of statistics of scattered light in comparison with the case of phonons in the number state. This qualitative difference can be used for experimental observation of squeezing of the vibrational
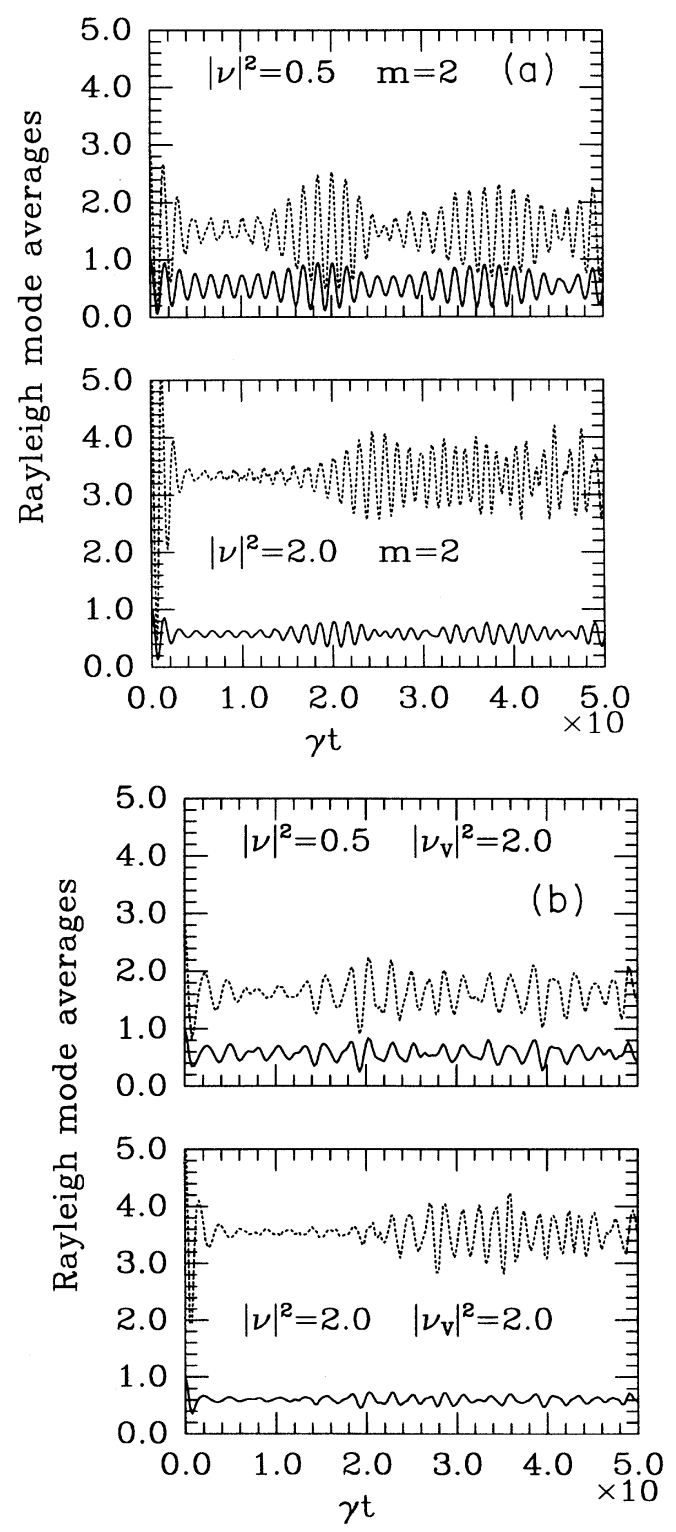

FIG. 4. Same as Fig. 1 for the Rayleigh mode initially in the squeezed state characterized by (a) $|\nu|^{2}=0.5$ (upper) and $|\nu|^{2}=2$ (lower) photons and $m=2$ phonons; (b) $|\nu|^{2}=0.5$ (upper) and $|\nu|^{2}=2$ (lower) photons and in the squeezed state phonons with $\left|\nu_{V}\right|^{2}=2$. 
mode.

It is possible to see from Fig. 1 the collapse-revival phenomenon occurring for initially squeezed phonon states. Qualitatively similar time dependent behavior is observed for the Stokes mode averages. The number oscillations for the Stokes photons have the mirror symmetry relative to the Rayleigh mode because of the ManleyRowe law [given in Eq. (4)] while the oscillations of the variance in number of photons strictly coincide with the corresponding Rayleigh mode variance. Such a mirror symmetry is a general property of a system independent of the initial state.

The dynamics of the system in initial coherent state of the Rayleigh mode is presented in Figs. 2 and 3 for the Rayleigh and Stokes modes, respectively. When the mean number of initial Rayleigh photons $\left\langle a^{\dagger} a\right\rangle_{0}=|\alpha|^{2}$ is small enough the distribution remains Poissonian for $t>0$, as the number of phonons in the vibrational mode $m$ increases. On the other hand, as $|\alpha|^{2}$ increases for a fixed $m$, a super-Poissonian statistics for the Rayleigh photons is observed. Similar conclusions may be drawn for the Stokes photons, although the oscillations in the variance do not coincide with those for Rayleigh photons, in this case. If the vibration mode is initially in a squeezed state, the response of the system is qualitatively similar [see Fig. 2(b)]. In Fig. 3 we show only the dynamics of the Stokes mode when the vibration mode phonons are initially in the squeezed state, since the case with phonons in the number state has a mirror symmetry to the ones shown in Fig. 2, similar to the discussion of Fig. 1.

Finally, we show the time dependence of the Rayleigh mode fluctuations in Fig.4, when the initial state of Rayleigh photons is a squeezed state. The main result we obtain from these figures is that the sub-Poissonian distribution is absent here, in contrast to the previous cases.
As for a brief summary, we list below some of the main conclusions of this work.

(1) The collapse-revival phenomenon is the property of the model under consideration, independent of the type of the initial state used to prepare the system (for the Rayleigh mode in the number state, it can be observed for $n>2$ ).

(2) The sub-Poissonian statistics is seen to be obeyed for the number and coherent initial state of the Rayleigh mode, but not for the squeezed vacuum state.

(3) The behavior of the scattered light in the number state differs qualitatively depending on the initial state of the vibration mode.

In connection with the last result, we note that the number states of the vibration mode may be considered as a state of harmonic phonons at zero temperature while the squeezed vacuum state corresponds to the correlated phonons due to some mechanism of interaction [2]. We can assume the number of initial phonons (or mean number) to be given. Then, the change in initial intensity of the Rayleigh mode and the observation of the corresponding change in the Mandel's factor

$$
Q=\frac{v_{t}\left(c^{\dagger} c\right)-\left\langle c^{\dagger} c\right\rangle_{t}}{\left\langle c^{\dagger} c\right\rangle_{t}}
$$

would allow us to find the type of phonon distribution present in the system.

\section{ACKNOWLEDGMENTS}

A.S. acknowledges the hospitality of the Physics Department at Bilkent University during his stay. A.S. also would like to thank Professors C. Bowden, R. Bullough, S. Carusotto, F. Persico, and V. Rupasov for fruitful discussions.
[1] Y. R. Shen, The Principles of Nonlinear Optics (Wiley, New York, 1984).

[2] A. S. Shumovsky, in Modern Nonlinear Optics, Part 2, edited by M. W. Evans (Wiley, New York, 1993).

[3] B. Crosignani, P. Di Porto, and M. Bertolotti, Statistical Properties of Scattered Light (Academic, New York, 1975).

[4] D. F. Walls, Z. Phys. 237, 224 (1970).

[5] B. R. Mollow and R. J. Glauber, Phys. Rev. 160, 1076 (1967).

[6] R. Graham, Z. Phys. 211, 469 (1968).

[7] F. Persico and G. Vetri, Phys. Rev. A 12, 2083 (1975).

[8] K. J. McNeil and C. W. Gardiner, Phys. Rev. A 28, 1560 (1983).

[9] W. J. Mielniczuk, Opt. Acta 26, 1115 (1979).

[10] H. P. Yuen, Phys. Rev. A 13, 2226 (1976).

[11] N. N. Bogolubov, J. Phys. (Moscow) 11, 23 (1947).

[12] G. P. Agarval and S. S. Iha, Z. Phys. B 25, 391 (1979).

[13] V. I. Rupasov and V. I. Yudson, Zh. Eksp. Teor. Fiz. 87, 1617 (1984) [Sov. Phys. JETP 60, 927 (1984)].

[14] A. S. Shumovsky, R. Tana's, and Tran Quang, Opt. Commun. 64, 45 (1987).

[15] A. S. Shumovsky and Tran Quang, in Interaction of
Electromagnetic Field with Condensed Matter, edited by N. N. Bogolubov, A. S. Shumovsky, and V. I. Yukalov (World Scientific, Singapore, 1990).

[16] C. C. Gerry and J. H. Eberly, Phys. Rev. A 42, 6805 (1990).

[17] D. F. Walls and C. T. Tindle, J. Phys. A 5, 534 (1972).

[18] J. Mostowski and K. Rzazewski, Phys. Lett. 66A, 275 (1978).

[19] J. Katriel and D. G. Hammer, J. Phys. A 14, 1211 (1981).

[20] P. Szlachetka, S. Kielich, J. Perina, and V. Perinova, Opt. Acta 27, 1609 (1980).

[21] S. Carusotto, Phys. Rev. A 40, 1848 (1989).

[22] Yu. Orlov, I. Pavlotsky, A. Shumovsky, V. Suslin, and V. Vedenyapin, Int. J. Mod. Phys. B. (to be published)

[23] Yu. N. Orlov and V. V. Vedenyapin, Mod. Phys. Lett. B (to be published).

[24] R. Bellman, Introduction to Matrix Analysis (McGrawHill, New York, 1960).

[25] G. Drobny and I. Jex, Phys. Rev. A 46, 499 (1992).

[26] H. I. Yoo and J. H. Eberly, Phys. Rep. 118, 239 (1985).

[27] Fam Le Kien and A. S. Shumovsky, Int. J. Mod. Phys. B 5, 2287 (1991). 